\title{
Genomic Variability of Phytophthora palmivora Isolates from Different Oil Palm Cultivation Regions in Colombia
}

\author{
Juanita Gil,1,2,4 Mariana Herrera, ${ }^{1}$ Jorge Duitama, ${ }^{2}$ Greicy Sarria, ${ }^{3}$ Silvia Restrepo, ${ }^{4}$ and Hernán Mauricio Romero ${ }^{1,5, \dagger}$ \\ ${ }^{1}$ Biology and Breeding Program, Colombian Oil Palm Research Center, Cenipalma, Calle 98 No. 70-91, Piso 14, 111121, Bogotá, Colombia \\ 2 Systems and Computing Department, Universidad de Los Andes, Carrera 1 No. 18A-12, 111711, Bogotá, Colombia \\ ${ }^{3}$ Pests and Diseases Program, Colombian Oil Palm Research Center, Cenipalma, Calle 98 No. 70-91, Piso 14, 111121, Bogotá, Colombia \\ ${ }^{4}$ Biological Sciences Department, Universidad de Los Andes, Carrera 1 No. 18A-12, 111711, Bogotá, Colombia \\ ${ }^{5}$ Department of Biology, Universidad Nacional de Colombia, Carrera 45 No. 26-85, 111321, Bogotá, DC, Colombia \\ Accepted for publication 18 April 2020.
}

\begin{abstract}
Palm oil is the most consumed vegetable oil globally, and Colombia is the largest palm oil producer in South America and fourth worldwide. However, oil palm plantations in Colombia are affected by bud rot disease caused by the oomycete Phytophthora palmivora, leading to significant economic losses. Infection processes by plant pathogens involve the secretion of effector molecules, which alter the functioning or structure of host cells. Current long-read sequencing technologies provide the information needed to produce high-quality genome assemblies, enabling a comprehensive annotation of effectors. Here, we describe the development of genomic resources for P. palmivora, including a high-quality genome assembly based on long and short-read sequencing data, intraspecies variability for 12 isolates from different oil palm cultivation regions in Colombia, and a catalog of over 1,000 candidate effector proteins. A total of 45,416 genes were annotated
\end{abstract}

ABSTRACT from the new genome assembled in 2,322 contigs adding to $165.5 \mathrm{Mbp}$, which represents an improvement of two times more gene models, 33 times better contiguity, and 11 times less fragmentation compared with currently available genomic resources for the species. Analysis of nucleotide evolution in paralogs suggests a recent whole-genome duplication event. Genetic differences were identified among isolates showing variable virulence levels. We expect that these novel genomic resources contribute to the characterization of the species and the understanding of the interaction of P. palmivora with oil palm and could be further exploited as tools for the development of effective strategies for disease control.

Keywords: allelic variants, genetics and resistance, genome assembly, mycology, techniques
The African oil palm (Elaeis guineensis Jacq.) is the most cultivated species for vegetable oil production worldwide and the primary source of edible oil exceeding soybean and other crops due to its higher yield and quality (Teixeira da Silva and Engelmann 2017). Colombia has the largest palm oil production in South America, and it is the fourth largest producer worldwide after Indonesia, Malaysia, and Thailand (GreenPalm 2019). In 2017, oil palm plantations covered around 412,076 ha of land, producing more than 1.6 million tons of oil per year (Fedepalma 2018). Oil palm plantations are distributed in four zones of the country: North, Center, East, and Southwest (Castiblanco et al. 2013).

One of the major constraints limiting palm oil production in Colombia is bud rot disease caused by the oomycete Phytophthora palmivora (E.J. Butler) E.J. Butler (Sarria et al. 2008; Torres et al. 2010). This disease has caused the destruction of more than

${ }^{\dagger}$ Corresponding author: H. M. Romero; hmromeroa@unal.edu.co

Current address of J. Gil: Department of Entomology and Plant Pathology, University of Arkansas, 72701, Fayetteville, AR, U.S.A.

Funding: This project was funded by the Departamento Administrativo de Ciencia, Tecnología e Innovación (Colciencias Contract 158-2016) and the Colombian Oil Palm Promotion Fund (FFP) administered by Fedepalma (2016-2019). This project is authorized by Access to Genetic Resources Contract 194-2017 between Cenipalma and the Environmental Ministry of Colombia. The isolates of $P$. palmivora are Colombian biological resources.

*The $e$-Xtra logo stands for "electronic extra" and indicates that supplementary figures, six supplementary tables, and two supplementary scripts are published online.

The author(s) declare no conflict of interest.

(c) 2020 The American Phytopathological Society
35,000 ha and the loss of U.S. \$250 million in the Central region alone (Torres et al. 2016). Within the oomycetes, Phytophthora species are the most commonly studied due to their economic importance, because many different crops are affected by this genus, leading to annual losses of billions of dollars (Kamoun et al. 2015). $P$. palmivora is an important species of the genus because it infects a wide variety of hosts and tissues, causing diverse diseases (Drenth and Guest 2004). This capacity to attack different hosts makes P. palmivora an important species to study plant-pathogen interactions and complex disease cycles (Drenth and Guest 2004). Besides oil palm, $P$. palmivora has been identified on crops such as cocoa, papaya, rubber, coconut, black pepper, durian, and barley, causing root, bud, and rotting diseases (Drenth and Guest 2004; Le Fevre et al. 2016). Bud rot disease in oil palm has been observed since 1960 in Colombia, but the characterization of $P$. palmivora as the causal agent is very recent (Sarria et al. 2008; Torres et al. 2010). This late characterization has not only delayed useful research on the pathogen but also hindered the development of effective strategies on how to control the disease (Torres et al. 2016).

The infection of oil palm with $P$. palmivora is so destructive because the pathogen attacks the leaflets near the heart of the palm, causing necrosis. If favorable conditions for disease development prevail and the spear leaf opens, then sporangia and zoospores might be released, which are carried toward the base of the spear leaf causing more lesions that lead to the destruction of young developing tissue and the meristem (Torres et al. 2016). P. palmivora shows a hemibiotrophic lifestyle, typical of the genus Phytophthora (Adhikari et al. 2013). The biotrophic phase is characterized by the formation of appressoria and haustoria to enter and colonize the host tissue without causing disease symptoms. During the necrotrophic phase of the life cycle, necrosis of the tissue is induced to acquire nutrients more efficiently and produce 
sporangia to release zoospores (Le Fevre et al. 2016). Transcriptomic studies in Nicotiana benthamiana have shown that within $24 \mathrm{~h}$ after inoculation (hai), the biotrophic stage is established, corresponding to an early infection phase. Then, between 48 to 72 hai, the necrotrophic stage happens, which corresponds to a late infection phase. The switch between the two stages was observed at 30 hai (Evangelisti et al. 2017). Necrotic lesions on oil palm leaves are observed 2 days after inoculation, which corresponds to observations in other models of infection with P. palmivora (Mohamed Azni et al. 2017).

Plant pathogens have developed different infection strategies to evade the host immune system. The first line of immunity is through the recognition of conserved molecular signals, namely MAMPs or PAMPs (microbe- or pathogen-associated molecular patterns). If the pathogen overcomes this first barrier, then it usually releases effectors to block further defense mechanisms. Effectors are molecules secreted by pathogens which alter the functioning or structure of host cells to promote their development (Win et al. 2012). In oomycetes, effectors can be predicted by conserved motifs in the N-terminal region that follows the signal peptide, such as those found in RxLR and CRN type effectors. While RxLR effectors are representative of Phytophthora species, CRN effectors are found in all oomycetes (Bozkurt et al. 2012).

Researchers at the Colombian oil palm research center, Cenipalma, have investigated and analyzed bud rot disease for several years, finding that the development of symptoms and the plant responses to treatment and recovery after infection are variable between different regions of the country. Because the commercially cultivated palms are the same in all regions, it is suggested that the observed differences might be due to the region of cultivation and the pathogen, indicating a genotype-byenvironment interaction or pathogen variability. Hence, we believe that the identification of effector proteins in P. palmivora, as well as an understanding of the variability of the pathogen in Colombia, could provide broader knowledge about the host-pathogen interaction and contribute to the development of effective regional strategies for disease control. In this work, we aimed to characterize the genome of $P$. palmivora from oil palm and provide high-quality genomic resources that help to understand the diversity of the pathogen in a group of isolates from Colombia.

\section{MATERIALS AND METHODS}

P. palmivora isolates. Isolates selected for sequencing were collected at four different oil palm plantations in Colombia, corresponding to four different zones in the country, which vary in altitude, temperature, and humidity. Twelve isolates were selected and coded according to their region of origin as follows: four isolates (ZC01, ZC02, ZC03, and ZC04) from Puerto Wilches, Santander (latitude $7^{\circ} 20^{\prime} 54^{\prime \prime} \mathrm{N}$; longitude $73^{\circ} 53^{\prime} 54^{\prime \prime} \mathrm{W}$ ), three isolates (ZN01, ZN02, and ZN03) from El Retén, Magdalena (latitude $10^{\circ} 36^{\prime} 38^{\prime \prime} \mathrm{N}$; longitude $74^{\circ} 16^{\prime} 06^{\prime \prime} \mathrm{W}$ ), one isolate (ZOR01) from Cabuyaro, Meta (latitude $4^{\circ} 17^{\prime} 09^{\prime \prime} \mathrm{N}$; longitude $72^{\circ} 47^{\prime} 29^{\prime \prime} \mathrm{W}$ ), and four isolates (ZOC01, ZOC02, ZOC03, and ZOC04) from Tumaco, Nariño (latitude $1^{\circ} 48^{\prime} 24^{\prime \prime} \mathrm{N}$; longitude $78^{\circ} 45^{\prime} 53^{\prime \prime} \mathrm{W}$ ). A list of all sequenced isolates of $P$. palmivora and their origins is provided in Supplementary Table $\mathrm{S} 1$.

DNA extraction and sequencing. Isolate $\mathrm{ZC} 01$ was selected for long-read sequencing and de novo assembly using Illumina (Illumina, Inc., San Diego, CA) and PacBio (Pacific Biosciences of California, Inc.) platforms, and it is the isolate for which the reference genome was assembled. All other isolates were sequenced only through the Illumina platform. High-quality DNA for PacBio sequencing was obtained using the GeneJET Plant Genomic DNA Purification Mini Kit (catalogue number K0792) from Thermo Fisher Scientific (Waltham, MA). DNA for Illumina sequencing was obtained from $5 \mathrm{~g}$ of mycelium following the DNA extraction protocol by Goodwin et al. (1992). The obtained DNA was cleaned using the DNeasy PowerClean CleanUp Kit (catalogue number 12877-50) from Qiagen following the manufacturer's instructions. DNA quality was assessed by agarose gel electrophoresis using a $1 \%$ gel (TAE $1 \times)$, and by spectrophotometry, obtaining 260:280 ratios between 1.7 and 1.9 .

Library preparation and sequencing were performed by GenoHub (Austin, TX). Paired-end libraries for 150-bp read length were designed for Illumina sequencing on a HiSeq 2000 machine, while the PacBio libraries were designed to obtain $50 \times$ sequencing depth and sequenced on a PacBio Sequel instrument.

Reference genome assembly and annotation. The PacBio long reads were assembled de novo using Canu v1.7 (Koren et al. 2017) with the following parameters modified from the default settings: genomeSize $=105 \mathrm{~m}$, correctedErrorRate $=0.040$, and corMhapSensitivity $=$ normal. Other parameters were as default including rawErrorRate $=0.300$, minReadLength $=1,000$, and minOverlapLength $=500$. Illumina reads, corresponding to the same isolate, were aligned to this reference assembly to perform polishing and to validate the accuracy of the assembly. The polishing was performed using NGSEP v3.3.0 with the option VCFIndividualGenomeBuilder (Tello et al. 2019a). The alignment of the Illumina reads was carried out with bowtie2 v2.3.4.1 (Langmead and Salzberg 2013), allowing up to three alignments per read to identify unique, primary, and secondary alignments. Alignments were sorted by reference coordinates using picard (https://broadinstitute.github.io/picard/). Contigs with abnormal coverage were identified using samtools depth (Li et al. 2009).

Following the assembly of the reference genome, its structural annotation was performed using the MAKER pipeline (Cantarel et al. 2008). Genes were predicted ab initio using AUGUSTUS v.3.0.3. For the evidence-driven gene annotation, we integrated into the pipeline transcripts from an RNA sequencing experiment for the reference isolate described in Avila-Mendez et al. (2019), which had been de novo assembled with Trinity v.2.6.6 (Grabherr et al. 2011). Also, transcript and protein sequences from $P$. palmivora strain SBR112.9 obtained from NCBI (BioSample: SAMN04632378) were integrated. Accession IDs and URLs to download the datasets are available in Supplementary Table S2, including the raw data for the isolates sequenced in this study.

Functional annotation of the genome was performed using the suite Trinotate v3.1.1 (Bryant et al. 2017). Trinotate was run starting from reference guided transcript assemblies from Stringtie (Pertea et al. 2015). Custom scripts aided the integration of the results from the functional annotated transcriptome with the gene models annotated by MAKER. The complete assembly and annotation procedure is provided as Supplementary Script S1.

Completeness of the $P$. palmivora reference genome assembly and annotation were assessed by benchmarking nearly universal single-copy orthologs (BUSCO) (Simão et al. 2015). For this, the dataset of the Alveolata and Stramenopiles (https://busco.ezlab.org/), which contains 234 genes and 24 species, three of them belonging to the genus Phytophthora, namely $P$. infestans, $P$. ramorum, and $P$. sojae, was used.

The assessment of paralogs and orthologs within and between the genomes of $P$. palmivora (this study and public strain SBR112.9), as well as $P$. megakarya strain ZDHO120 and $P$. sojae V3.0 (strain P6497) was done using the module GenomesAligner from NGSEP v3.3.2 (Tello et al. 2019b), by which pairwise alignments of complete genomes are performed. To identify paralogs, only one genome and its corresponding annotation file are provided at a time. For genomes with sufficiently large N50 contig length (namely the assembly in this work and that of $P$. sojae) paralog pairs within the same contig and in different contigs were analyzed separately. For the latter case, a custom script was used to verify if at least half of 10 genes surrounding each source gene have a consistent paralog in the target contig, showing then some evidence of local synteny (Supplementary Script S2). We selected 5,619 paralog pairs showing this pattern in our assembly, which was much higher than 
the number of pairs obtained for $P$. sojae (147), despite the much better contiguity of the $P$. sojae genome ( 82 scaffolds). We finally calculated statistics of nucleotide evolution for each paralog and ortholog pair (namely the ratio Ks of synonymous substitutions and the $\mathrm{Ka} / \mathrm{Ks}$ ratio of nonsynonymous to synonymous substitutions (Kimura 1977). For each homolog pair, pairwise alignment of the CDS nucleotide sequences consistent with the codon organization was performed (class ngsep.transcriptome.CodonCDSPairwiseAlignment of NGSEP). Then, the codeml function of PAML v4 was used to estimate nucleotide diversity values, using the model with two or more $\mathrm{dN} / \mathrm{dS}$ ratios for branches and the F3X4 table for codon frequencies (Yang 2007).

Ploidy estimation of $\boldsymbol{P}$. palmivora. The ploidy of the $P$. palmivora isolates obtained from oil palm was determined based on two different approaches implemented in NGSEP v3.3.0 (Tello et al. 2019a) and nQuire (Weiß et al. 2018). The first estimates the ploidy based on allele frequencies for sites showing base calls for more than one nucleotide from the alignment files. The second also calculates base frequencies for biallelic sites from the read alignments, but it additionally implements a Gaussian mixture model to model those frequencies and maximizes the likelihood of each calculated model using an expectation-maximization algorithm. Ploidy is finally estimated by calculating the delta loglikelihood between a free and each of the three fixed models (for diploidy, triploidy, and tetraploidy), where the smallest value indicates the most likely ploidy.

Genetic variability. After mapping the raw reads of each of the samples to the reference genome assembly and sorting the alignments by their genomic location, single nucleotide polymorphisms (SNPs) were called in every sample using the FindVariants module of NGSEP v3.3.0 (Tello et al. 2019a). Furthermore, the options -runRep -runRD -runRP -maxAlnsPerStartPos 5 -knownSTRs -minMQ 40 and -maxBaseQS 30 were used to assess genetic variability among the isolates. Detected variants were merged into one list to genotype them in each of the samples, after which the vcf files per sample were merged into a single one containing the list of all variable sites and the corresponding genotypes in the genomes of all samples. The final file was filtered to keep only biallelic SNVs with a minimum genotyping quality score of 40, variants that were genotyped in at least 11 of the 12 samples and a maximum observed heterozygosity of 0.8 over the samples.

Furthermore, repetitive regions and sites in which only one allele was observed were filtered out. Using the filtered variants file as input, we ran the module VCFDistanceMatrixCalculator from NGSEP v3.3.0 with default options. Based on the obtained matrix of genetic distances, inferences about the genomic differences between the isolates were established by neighbor-joining clustering.

To assess whether the Colombian $P$. palmivora isolates undergo sexual or clonal reproduction, the $\mathrm{R}$ package poppr (Kamvar et al. 2014) was used. The filtered vcf file was transformed into the STRUCTURE data format using the option ConvertVCF from NGSEP. The file was imported in $\mathrm{R}$ with the function import2genind(), and the function poppr() was subsequently used on the data.

Plant inoculations. Detached-leaf observations of disease progress were made for 10 of the 12 P. palmivora isolates, following the methodology proposed by Sarria et al. (2016), to elucidate some of the genetic differences seen in the oil palm pathogenic isolates. Immature oil palm leaflets were inoculated with $20 \mu \mathrm{l}$ of inoculum suspension at a concentration of 20,000 zoospores $/ \mathrm{ml}$ and maintained at 26 to $27^{\circ} \mathrm{C}$. Meanwhile, the Control leaflets were treated with sterile distilled water. The size of the lesion was measured 48, 72, and 96 hai. Six leaflets were placed on aluminum containers and inoculated per isolate. Six containers with six leaflets each were arrayed six times for each isolate. The lesion size was measured lengthwise using digital calipers (Supplementary Fig. S1). In total, 36 leaflets were evaluated for each isolate, and lesion sizes were averaged for each of the time points. Data at 96 hai were analyzed in a simple linear model and also performing a nonparametric test (Wilcoxon test) comparing every pair of samples with a significance level $\alpha$ of 0.05 and Bonferroni correction.

Identification of effector proteins. The reference sequence in fasta format, as well as the structural annotation of the genome (gff3 format), were used to predict the proteins in the reference genome assembly of $P$. palmivora. This proteome was used as input for the R library effectR (Tabima and Grünwald 2019) to predict candidate effectors. The list of candidates was obtained based on the search of the conserved motifs of the RxLR and CRN effectors by using regular expressions and also based on an expanded search by homology of the sequences using a multiple alignment of the proteins obtained with MAFFT (Nakamura et al. 2018) and HMMER v.3.2.1 (Eddy 2008). Then we validated the presence of a signal peptide in the sequences running signalP4.1 (Petersen et al. 2011) with default D-cutoff values for eukaryotes.

\section{RESULTS}

The genome of $\boldsymbol{P}$. palmivora is diploid, highly repetitive, and gene-rich. We achieved an assembly for $P$. palmivora in 2,322 contigs with NG50 of 220,825 bp and a total length of $165.5 \mathrm{Mbp}$. The initial number of PacBio reads was 923,648, with 7,180 Mbp sequenced in total. The average read length was $8,284 \mathrm{bp}$, and the overall final coverage generated for our assembly was $43.4 \times$. The use of long reads allowed the resolution of complex regions during genome assembly; the short but less error-prone reads supported the polishing process to correct errors and achieve high base pair quality. Repetitive regions in the genome of $P$. palmivora added to $148.8 \mathrm{Mbp}$, representing $89.9 \%$ of the genome. Contigs with abnormal coverage were identified as part of the genome assembly validation steps. Still, they were not removed because they mostly corresponded to large repetitive structures that are part of the genome. To validate this, a BLAST search of 30 randomly selected contigs with abnormal coverage delivered hits to other Phytophthora species, including complete mitochondrial genomes. Supplementary Table S3 reports the normalized coverage per contig, including the contigs with abnormal coverage.

The evaluation of the content of universal single-copy ortholog genes showed that the reference genome assembly had only 10 missing genes, which is $4.3 \%$ of the dataset, and three fragmented genes. The number of complete genes was 221, from these 126 are duplicated (53.8\%), and 95 are single copy (40.6\%) (Supplementary Fig. S2). Quality base-pair level of the assembly was assessed based on the overall alignment rate of Illumina reads from isolate ZC01 for which $98.8 \%$ of aligned reads was obtained. The final coverage of the Illumina assemblies of the other isolates ranged between $100.4 \times$ and $106.4 \times$ and is reported in Supplementary Table S4.

We annotated 45,416 gene models based on prediction ab initio and integrating evidence from RNA sequencing experiments (Avila-Mendez et al. 2019) and data of transcripts and proteins from P. palmivora strain SBR112.9. We assessed how many of those gene models were fully covered by transcripts, finding that 19,438 genes in the reference annotation were covered end to end by RNAseq reads. From the initially annotated gene models, 19,398 (44\%) have known functions and gene ontology (GO) terms associated, identified based on referenced guided assembled transcripts. Some of the most common functions after GO annotations, associated with at least 3\% of the genes, are shown in Supplementary Figure $\mathrm{S} 3$. The most common functions in biological processes are related to nitrogen metabolism and transport, and the most common molecular function is ion binding. However, $18.2 \%$ of the genes were associated with at least one of the functions related to enzyme activities and transmembrane transport, which might be necessary for pathogenicity. Regarding cellular component annotations, only a small number of genes were associated with extracellular regions $(<5 \%)$. 
Different statistics were calculated to assess the quality of the structural annotation. These results were compared with previous data for the species, obtained from the cacao isolate SBR112.9, and two other species to validate the quality of the annotation process. We calculated the annotated transcript and protein length, as well as the number of exons and size of the genes as measures of quality. All distributions exhibited the same trends as those obtained for the public annotation showing, however, a tendency toward larger transcripts, proteins, and genes in our annotation. This tendency was also observed in P. sojae, one of the best-assembled Phytophthora genomes available on NCBI, that also showed a larger number of genes with sizes above 7,000 bp, demonstrating that genes of these sizes are not abnormal for the genus (Fig. 1). For example, the largest gene annotated for our reference isolate is $10,377 \mathrm{bp}$ long. It has a Fibronectin type III domain that is not found in the annotation file for P. palmivora strain SBR112.9. The same was true for other of the larger genes $(>7,000 \mathrm{bp})$ with functional annotations not represented in the annotation of strain SBR112.9. In general, around $90 \%$ of the transcripts and genes in P. palmivora were equal to or smaller than 2,000 nucleotides, and most of the proteins $(95.5 \%)$ were smaller than 800 amino acids. Regarding the number of exons per gene, most of the genes had up to three exons. Although the distribution of sizes is similar, we were able to retain more valid transcripts (considering the identification of start and stop codons in the sequences) compared with strain SBR112.9 (91.1\% versus $80 \%$ ).

We also evaluated the number of paralog genes in our genome assembly. We compared it with the number of paralogs found in the public genome assembly for $P$. palmivora strain SBR112.9, as well as with the number of paralogs found in the genomes of
P. megakarya strain ZDHO120 (Ali et al. 2017) and P. sojae V3.0. While only $12.6 \%$ of the genes had a paralog in $P$. megakarya, we found that $41 \%$ (P. palmivora SBR112.9) and $77.7 \%$ (P. palmivora assembly from the present study) had at least one paralog. The number of paralogs in the genome of $P$. sojae was also very low, and despite being a well-assembled genome, it was challenging to identify synteny blocks. We were able to identify small synteny blocks of paralogous genes in our genome assembly, as well as paralogs that are found in the same scaffold. This difference could not be obtained from the assembly of strain SBR112.9 due to its high fragmentation (Fig. 2A). We calculated the Ks distribution for the paralogous genes of the three species and for orthologs between the Colombian P. palmivora isolate ZC01 and the other Phytophthora strains. The Ks distribution of orthologs corresponds with the phylogeny of the family (Supplementary Fig. S4). Moreover, the number of paralogs with the evidence of synteny and the distribution of $\mathrm{Ks}$ in these paralogs suggests a recent wholegenome duplication event in the evolutionary history of $P$. palmivora.

The estimation of the ploidy of the reference and the other isolates of $P$. palmivora was performed based on two similar approaches. Both methods estimated that the $P$. palmivora isolates tested in this study are diploid. With nQuire we obtained the lowest delta loglikelihood value for the diploid model, which represents the most likely ploidy for the sample (data not shown). Figure 2B shows the results for all isolates of $P$. palmivora as the distribution of allele frequencies obtained with NGSEP. This distribution has two peaks. One representing raw base calling errors, which are the high counts at the lower values of the allele proportion, and the second one appears above 0.4 , which shows that at heterozygous sites, alleles

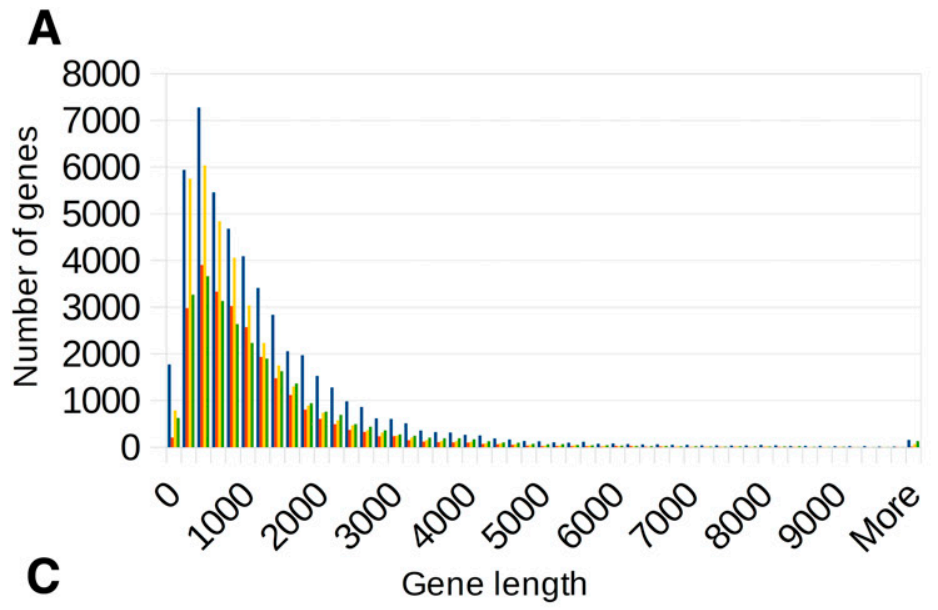

B
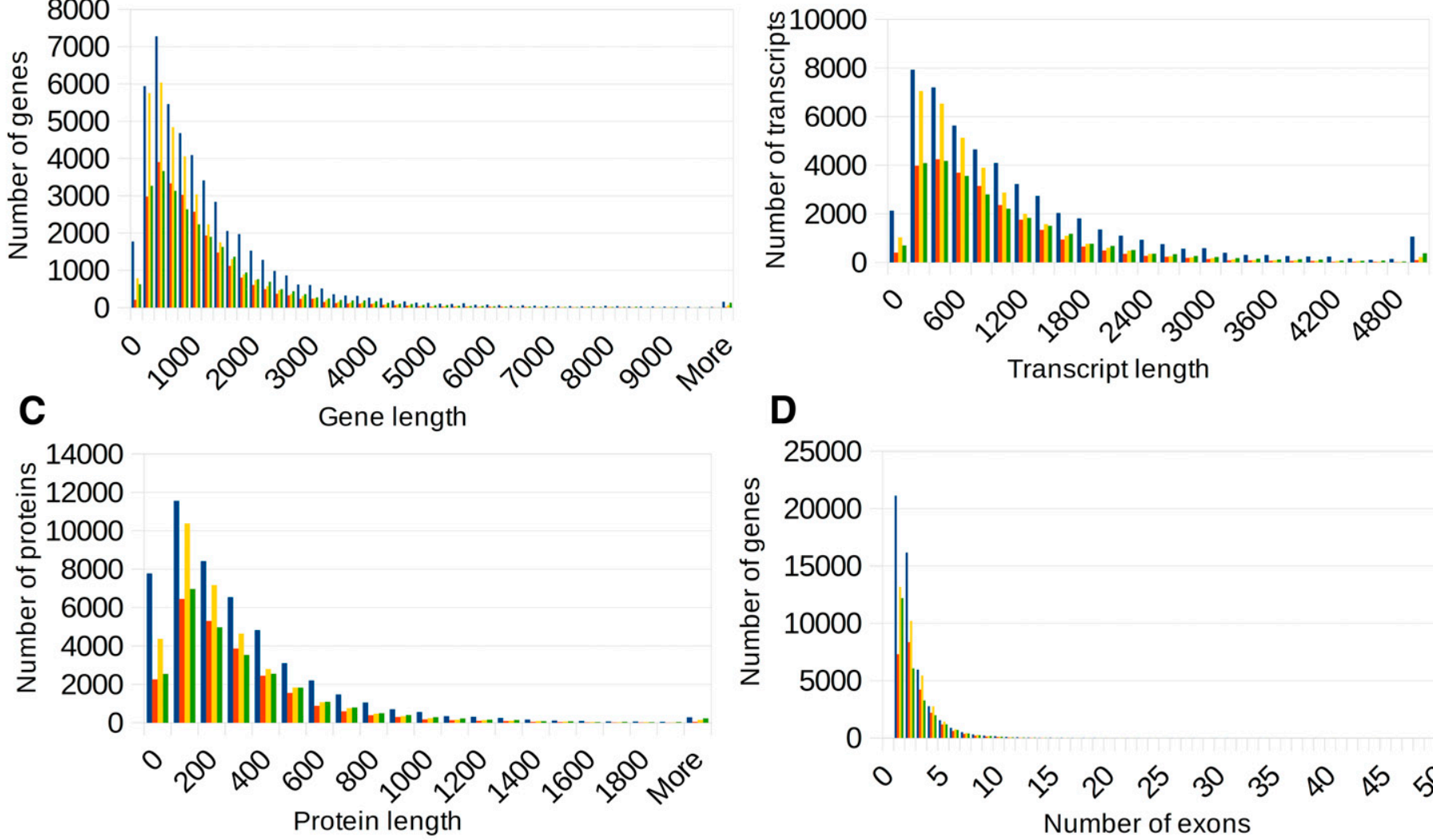

D

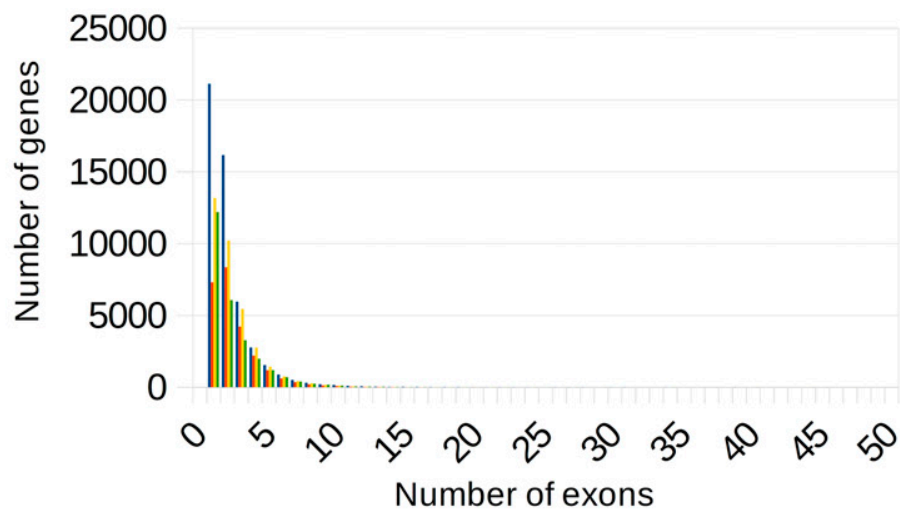

ZC01

SBR112.9

Pmeg — Psojae

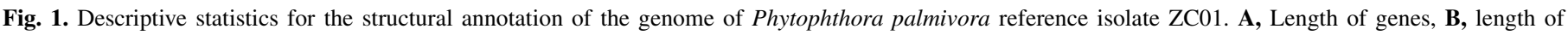

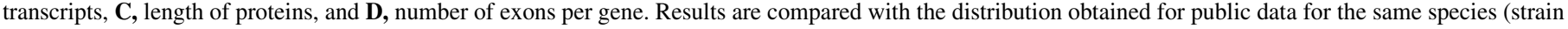
SBR112.9, red), and species P. megakarya (Pmeg, yellow) and P. sojae (Psojae, green). 
are found in a similar proportion, which is expected for diploid organisms.

High heterozygosity in the genome of $P$. palmivora, but low genetic diversity among Colombian isolates. The overall alignment rate of the raw Illumina reads of all isolates to the reference genome assembly was above $98 \%$ for 10 of the 12 samples. Isolates ZOC01 and ZOR01 had lower overall alignment rates, corresponding to 88.3 and $97.4 \%$, respectively (Supplementary Fig. S5). The total number of variants discovered in the samples was 221,196 . These variants were genotyped and then filtered as described in the Materials and Methods section. After filtering, the total amount of biallelic SNVs in the 12 samples was 1,279, from which 343 were located within coding regions. The most common variant annotation among the samples was the upstream transcript variant (537), followed by intergenic variants (288) and missense variants (135). The number of heterozygous sites per sample ranged from 104 (ZOC02) to 535 (ZOR01). All sites are reported in Supplementary Table S5 for each of the samples. We determined an average density of SNVs per kilobase pair of around 1.2 for $P$. palmivora in the whole genome in our samples, considering heterozygous and homozygous genotypes. This value corresponds to what has been determined for Phytophthora species such as $P$. infestans, which has a density of $0.65 \mathrm{SNPs} / \mathrm{kbp}$ on average between strains (Maurice et al. 2019).

From the filtered variants file, a distance matrix was calculated and used as input for a neighbor-joining approach to obtain a graphical overview of genetic distances (Fig. 3). From the dendrogram, it was observed that the isolates mostly clustered together. Two of the isolates, one from the Central region ( $\mathrm{ZC02}$ ) and the isolate from the East region (ZOR01), were separated from
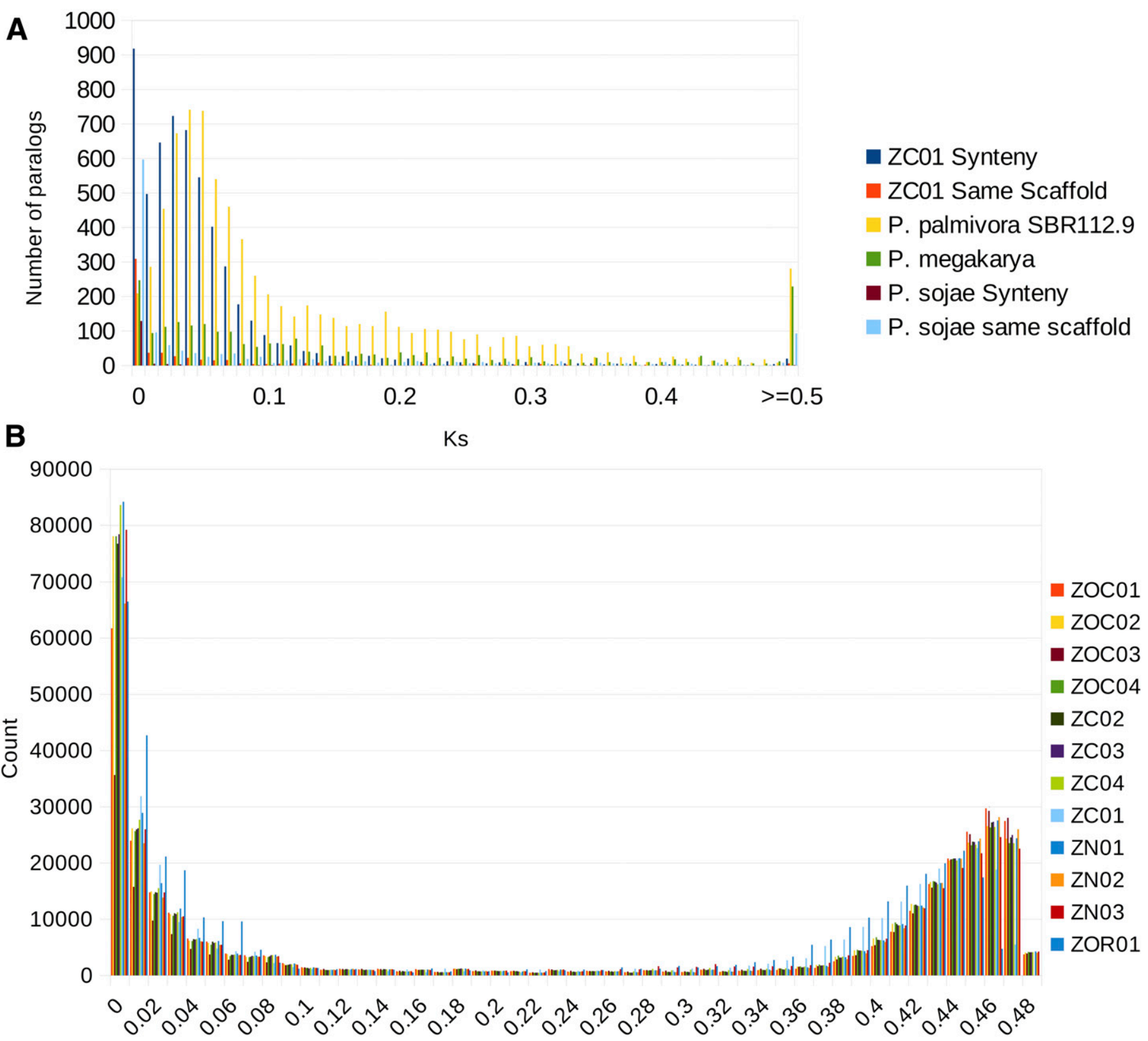

Allele proportion

Fig. 2. Analysis of the nucleotide evolution of Phytophthora palmivora. A, Number of paralogous genes in P. palmivora (ZC01 and SBR112.9), P. megakarya, and $P$. sojae. B, Distribution of allele frequencies for all isolates to estimate ploidy. Two peaks are observed: the peak at low values $(<0.1)$ represents raw base calling errors and the peak toward 0.5 represents diploidy, which means that at heterozygous sites the alleles are found in the same proportion. 
the others. Analyzing the variants identified in these samples, we found that these two samples had the largest amount of nonreference genotype calls (86\% of them heterozygous) with 611 and 618 SNPs for isolate ZC02 and ZOR01, respectively. While these variants represent around $50 \%$ of the entire catalog, the other samples only had between 100 and 200 nonreference genotype calls. These two samples segregated from the others by 332 SNPs, many of them being missense variants located mostly in hypothetical proteins. Isolates $\mathrm{ZC01}$ and $\mathrm{ZOC} 03$ also separated from the main cluster. However, they remained close to the other isolates. For these samples, we also observed a larger number of SNPs called as heterozygous genotypes compared with the samples that belong to the main cluster. A population analysis was performed to determine the reproductive strategy of the Colombian $P$. palmivora isolates. We obtained a standardized index of association $r$ (bar) $d=0.19(P<0.001)$. This value falls outside of the distribution expected under no linkage, indicating that alleles are linked across loci and that the population is clonal (Supplementary Fig. S6).

After assessing virulence of the isolates on detached leaves, it was noted that isolates ZC02 and ZOR01 showed a similar disease progression over time and the lesion sizes in the leaflets (96 hai) were smaller than the average lesion size considering all the isolates ( 26.5 and $25.4 \mathrm{~mm}$, respectively, average is $37.5 \mathrm{~mm}$ ). We also observed that one of the isolates from the North region (ZN01) caused the smallest lesions in the leaflets after $96 \mathrm{~h}$, being the least virulent among the isolates (Fig. 4). However, this isolate is within the main cluster in the dendrogram and thus grouped with the rest of the isolates, including three of the isolates from the Southwest region, which caused the most extensive lesions. When compared statistically, the differences in the average lesion size caused by isolates ZC02, ZC03, and ZOR01; ZN01 and ZOR01; ZC04 and ZOC01; ZNO2, ZNO3, ZOC01, and ZOC02; and ZOC02 and ZOC04 were not statistically significant, whereas the differences between the smallest lesion sizes (average below $29 \mathrm{~mm}$, observed for isolates $\mathrm{ZC02}, \mathrm{ZC03}, \mathrm{ZN01}$, and ZOR01) and the largest lesion sizes were statistically significant (Wilcoxon test, $P<0.001$ ).

A comprehensive catalog of predicted effector proteins for $\boldsymbol{P}$. palmivora. We identified over 1,400 candidate effectors in the genome of $P$. palmivora. Based on the functional annotation of the genome, namely domains identified by HMMER from the Pfam database, we initially identified some classes of putative

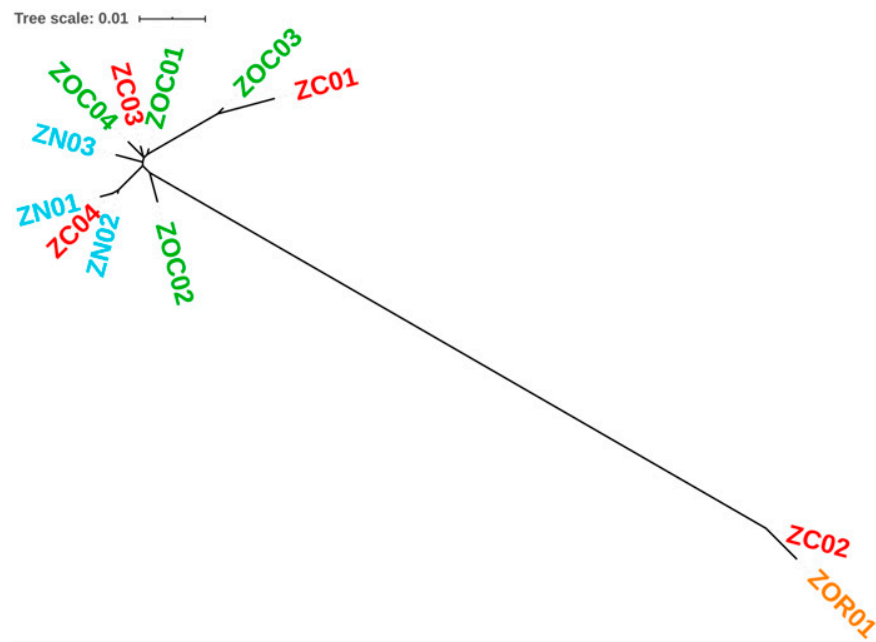

Fig. 3. Neighbor-joining dendrogram of distances between Phytophthora palmivora isolates from oil palm based on genome-wide high-quality single nucleotide polymorphism markers. Isolates are coded according to their region of origin followed by a consecutive number. ZC are samples from the Central region in Colombia, ZN from the North, ZOC from the Southwest, and ZOR from the East. extracellular and infection-related proteins (Fig. 5). From these, the kinases and repeat-containing proteins accounted for $15.6 \%$ of the Pfam counts. An important category within this subset is the effectors with 666 proteins (1.6\%), which are depicted in Figure 5B. By looking for the conserved motifs of the RxLR and CRN effectors in the protein sequences, we predicted 752 RxLR and 27 CRN candidate effectors. Following a more thorough search of candidate proteins by using profile hidden Markov models (HMMs) to retrieve sequence homologs from the sequence database, 623 new RxLR candidate effector proteins were identified, accounting for a total of 1,375. Regarding the CRN effectors, 95 new proteins were identified, making up 122 in total. The initial candidate effector proteins and their genomic location are listed in Supplementary Table S6. After analyzing the sequences for the presence of a signal peptide to consider them as secreted proteins, only 799 RxLR and eight CRN remained.

For the RxLR effectors, two conserved motifs were identified in the protein sequences between positions 48 and 68. These are the RxLR and the EER motifs. For the CRN we also identified two conserved motifs: one is the LFLAK motif at position 52 and the HVLV motif at position 108 of the protein sequences. A signal peptide was predicted for 580 of 913 complete RxLR candidate effectors (having both conserved motifs, RxLR and EER), which corresponds to $63.5 \%$. If considering only the RxLR candidate effectors retrieved after the first step of the effectR workflow (search of motifs by regular expressions), $77.4 \%$ of the complete proteins showed a signal peptide based on bioinformatics prediction, which represents 578 proteins out of 747 . The same analysis was performed for the $\mathrm{CRN}$ candidate effectors, where no signal peptide could be predicted in the complete candidate effectors (having the two conserved motifs, LFLAK and HVLV). Half of the identified CRN effectors with the LFLAK motif showed a signal peptide. Still, only for $4 \%$ of predicted effectors with the HVLV motif, a signal peptide was identified despite being the largest group within the CRNs (Fig. 6). The use of HMMs to search for homolog sequences allowed the identification of more candidate effectors having only one of the two conserved motifs but with the presence of signal peptides.

A multiple alignment of the sequences of the predicted RxLR effectors with both conserved motifs revealed four amino acids that most likely took the position of the $x$ amino acid in the motif: $F, L, S$, and R. The RFLR motif was present 176 times, the RLLR 151, RSLR 89, and RRLR 35 (Fig. 7). This shows the diversity within the RxLR effectors in the genome of $P$. palmivora.

\section{DISCUSSION}

Genomic approaches to study virulence mechanisms in the broad range of Phytophthora oomycetes have been limited by the difficulties related to the assembly of the highly repetitive genomes of these species. Recent advances in long-read sequencing technologies and bioinformatics have changed this scenario providing a large amount of genomic information between and within species. In this study, we achieved a high-quality assembly of the genome of the oil palm pathogen $P$. palmivora. This species belongs to clade 4 in the phylogenetic classification of the genus Phytophthora (Martin et al. 2014) consisting of three species: $P$. querceterum, an oak pathogen; $P$. megakarya, affecting cacao trees; and $P$. palmivora a multihost pathogen. The recently published genomes of $P$. megakarya and $P$. palmivora from cacao trees affected by black pod rot disease are highly fragmented as being assembled from short Illumina reads only (Ali et al. 2017). Here, we report a genome assembly of $165.5 \mathrm{Mbp}$ represented in 2,322 contigs and NG50 value of $220,825 \mathrm{bp}$, which is around 11 times less fragmented and 33 times more contiguous than the currently available sequence for $P$. palmivora. Moreover, $P$. palmivora is an important species not only within clade 4 but also within the genus, because it can affect multiple economically 
important crops worldwide. Thus, providing an improved assembly for a $P$. palmivora isolate obtained from oil palm is considerable progress for current and future research and represents a valuable genomic resource.

Possibly due to a better resolution of complex or repetitive regions for this genome assembly, we identified more gene structures than previously reported for this species, especially effectors and other infection-related genes. We obtained over 1,400 candidate effectors with the conserved motifs for the types RxLR and CRN (1,375 and 122, respectively). As recommended by
Tabima and Grünwald (2019), after identifying candidate effectors, these should be validated for having a signal peptide and therefore being classified as secreted proteins. Once the candidate sequences were subjected to the identification of signal peptides, the number of valid candidate effectors was considerably reduced (799 RxLR and eight $\mathrm{CRN}$ ). We compared the results we obtained with recent publications for the set of effectors of P. palmivora: Ali et al. (2017) identified 991 RxLR and 137 CRN effectors in total, but then only 414 and 4 RxLR and CRN, respectively with predicted signal peptide sequences. Evangelisti et al. (2017) reported 140 RxLR and
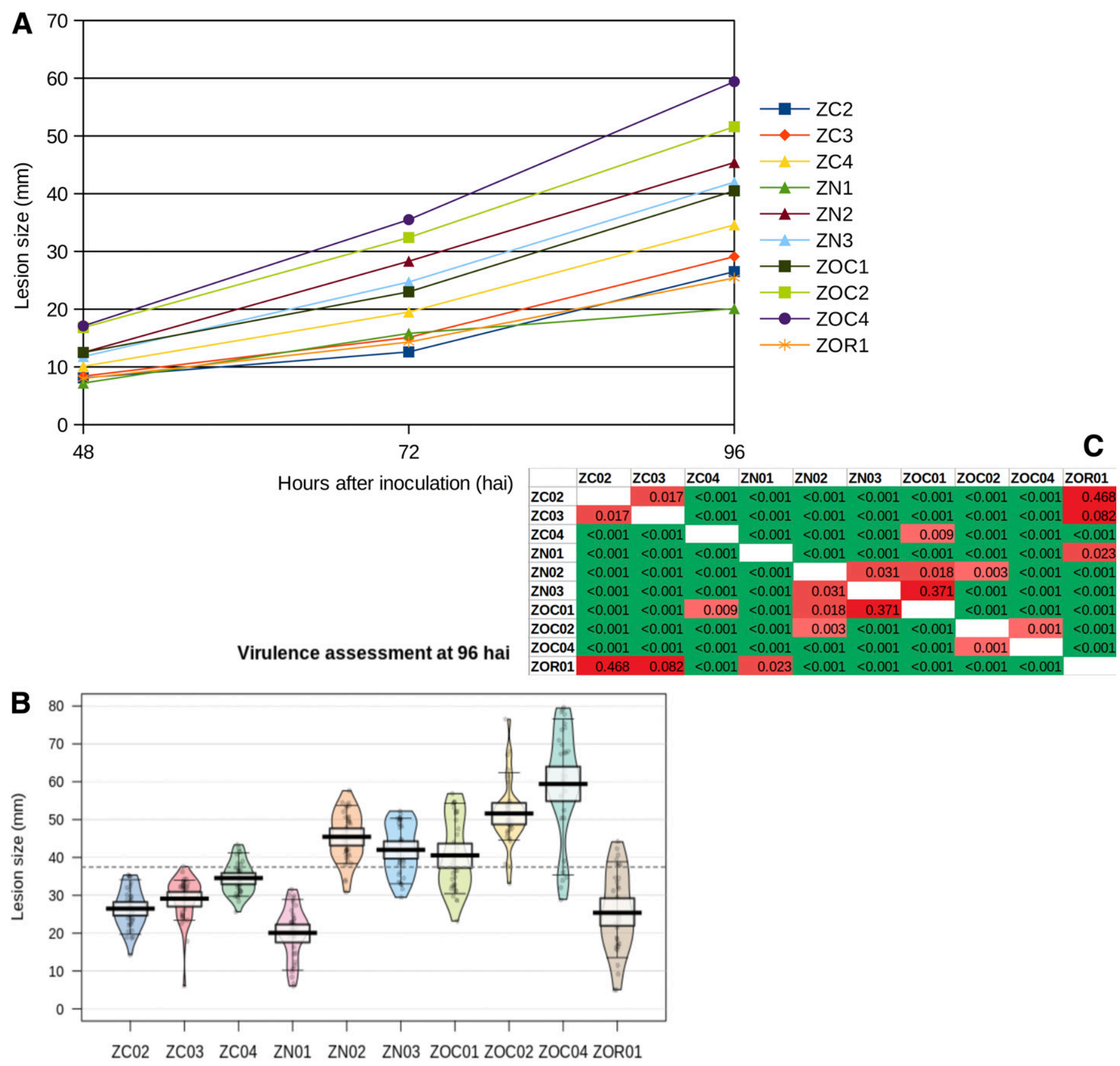

Phytophthora palmivora isolate

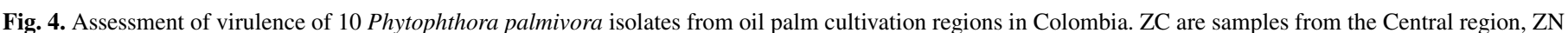

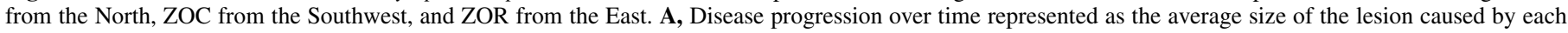

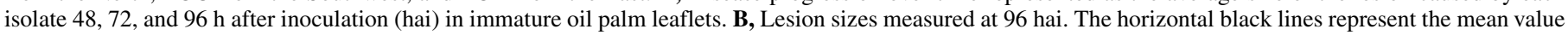

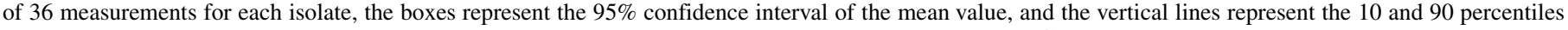

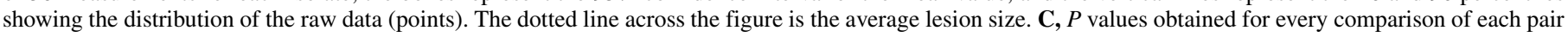

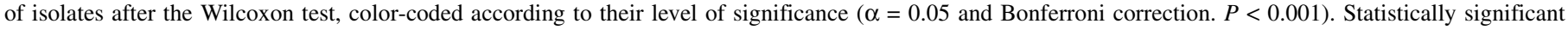
values are green. Not significant values are red. 
$15 \mathrm{CRN}$ from the predicted secretome based only on a de novo transcriptome assembly from a Colombian $P$. palmivora isolate (LILI) corresponding to ID ZOC01 in the present study. From the 15 CRN effectors, however, only three were identified initially as putative proteins. We based our prediction of effector proteins not only on transcriptomic data as in the two previously mentioned publications but also on a high-quality genome assembly and annotation. This approach integrated ab initio predicted genes and expression data from RNA-seq experiments of the interaction of the pathogen with oil palm (Avila-Mendez et al. 2019). In this way, a larger number of predicted effectors, especially for the RxLR effectors was considered.

Furthermore, potential infection-related genes were identified in greater representation from the annotated genes, such as cell wall degrading proteins and genes involved in detoxification metabolism. The expansion of these gene families in other Phytophthora species has been previously discussed (Yang et al. 2018). It gives a perspective for future studies on other less studied classes of effectors. Our results also promote the analysis of the evolution of effectors within the genus since we identified a great diversity within the predicted RxLR effectors.

By assessing the percentage of single-copy orthologs in the genome of $P$. palmivora, we recovered $95.7 \%$ of the genes from a dataset of 234 genes. Although these genes are considered nearuniversal single-copy orthologs, we found that $53.8 \%$ of them are duplicated in the genome of $P$. palmivora. The analysis of orthologs among $P$. palmivora isolate ZC01 and $P$. megakarya, $P$. sojae, and $P$. palmivora strain SBR112.9 confirmed the known phylogenetic relationships between the species. Thus, more orthologous genes and lower rates of nucleotide evolution were identified between the two $P$. palmivora isolates than against $P$. megakarya and $P$. sojae. On the other hand, the search for paralog genes within the genomes of P. palmivora, both the public assembly for strain SBR112.9 and our assembly, as well as $P$. megakarya and $P$. sojae showed that a larger

A

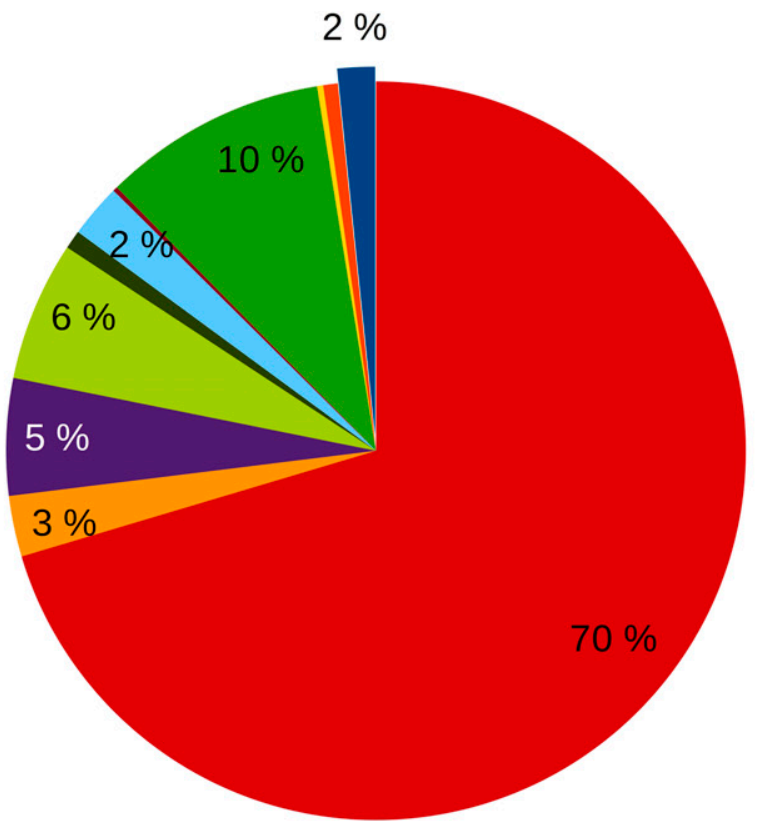

- Effectors and elicitors
- Proteases
Protease inhibitors
- Repeat-containing proteins
- Cysteine-rich proteins
- Cell wall-degrading proteins
- Oxidases
Kinases
- Detoxification metabolism
- Transposable elements
- Others

B

RXLR

NPP1

Elicitin

Jacalin-like lectin

Tgase elictor

RXLR WY-domain
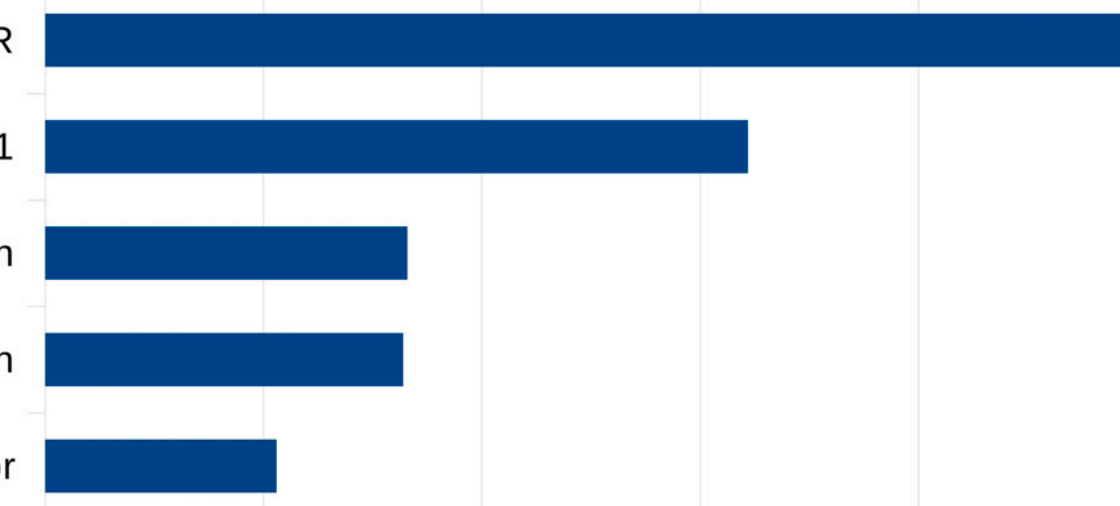

RXLR WY-domain

$0 \quad 50$

100

150

200

250

300

\section{Number of genes}

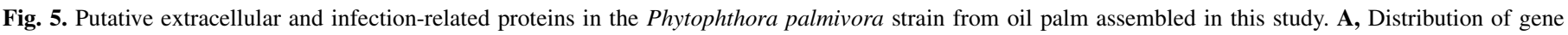

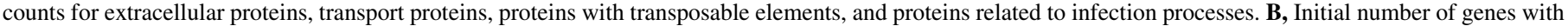
identified functional domains known to be related to effector proteins based on a Pfam database search. 
number of genes in $P$. palmivora had similarity to at least one other gene in its genome compared with the other two species. Having a less fragmented genome assembly allowed us to identify small synteny blocks and distinguish between paralogous genes in synteny from those that result from local duplications. Comparative analysis of nucleotide evolution of these synteny genes indicates a recent event of whole-genome duplication. The Ks distribution for paralogous genes revealed that $P$. palmivora isolates have a larger number of genes at Ks values of 0.03 , indicating a recent event of whole-genome duplication. This result supports the hypothesis by

A
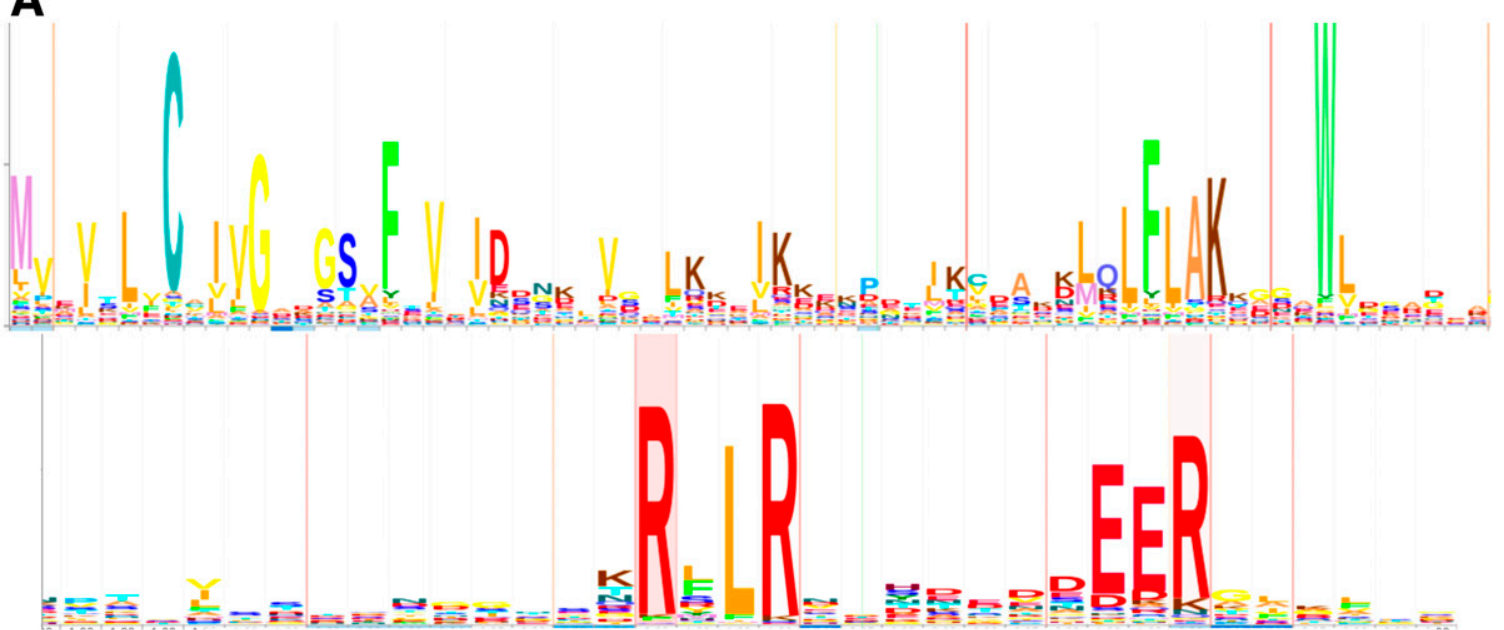

B

- effectR ш effectR only REGEX
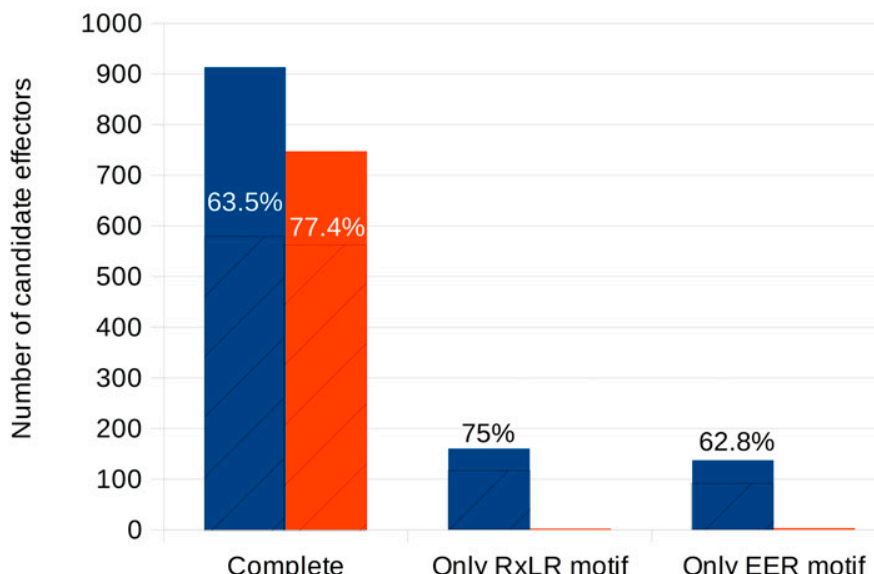

$7.9 \%$

Complete

Only RxLR motif

Only EER motif

No motif

C

a effectR a effectR only REGEX

60

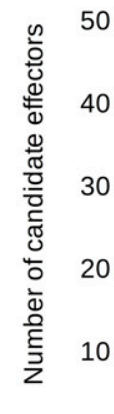

0
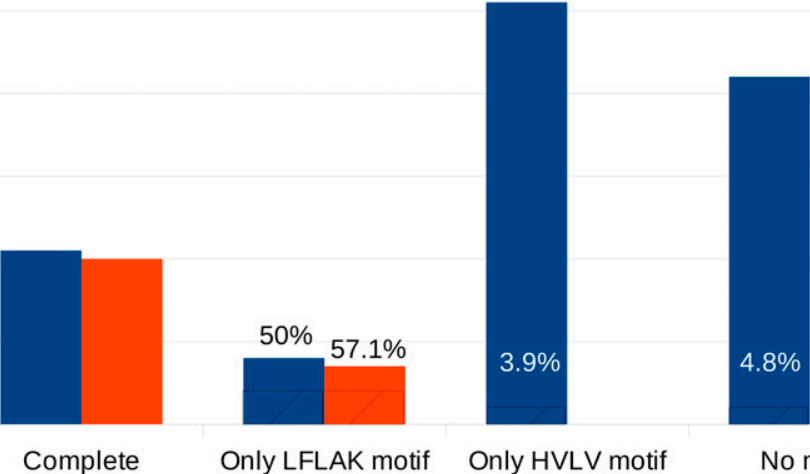

Complete

Only LFLAK motif

Only HVLV motif

No motif

Fig. 6. Predicted effectors from the proteome of Phytophthora palmivora reference isolate ZC01. A, Logo picture of aligned effector proteins showing conserved motifs in candidate effectors, CRN (upper panel) and RxLR. The conserved motifs for CRN are LFLAK and HVLV (not shown) and for RxLR are RxLR and EER. B, Number of RxLR effectors and C, CRN effectors predicted based on regular expressions and hidden-Markov models (HMMs) (blue bars) and only by regular expressions (red bars) using effectR. Effectors are divided into four categories, where complete means having both conserved motifs. The number of effectors with signal peptides are represented by the diagonal lines within the solid bars and the respective value expressed as percent values is shown above each bar. 
Ali et al. (2017) of a whole-genome duplication event in $P$. palmivora after speciation. It is consistent with the total assembly length, close to twice the assembly length for P. megakarya and $P$. sojae. However, it appears inconsistent with the analysis of ploidy for the $12 \mathrm{P}$. palmivora isolates collected from different oil palm cultivation regions in Colombia. This analysis, carried out by two different methods and based on relative allele counts for heterozygous sites, does not show a peak of heterozygous variants at $1: 3$ relative allele proportions and hence infers that the species is diploid. A possible explanation of this outcome is that, due to the normally clonal reproduction mode of the species, original chromosome quadruples evolved independently through mutation without recombination, and now chromosome pairing for meiosis behaves like that of a normal diploid individual. Several examples of similar nucleotide evolution patterns have been observed in plant species (Qiao et al. 2019). A chromosome-level assembly for $P$. palmivora should be performed to validate this hypothesis, integrating current scaffolding technologies such as the chromosome interaction mapping (Hi-C) (Bickhart et al. 2017).

Based on genetic distances calculated from high-quality biallelic SNVs identified in each sample, most of the $P$. palmivora isolates from oil palm in Colombia did not differ from each other. This result, together with the population analysis, suggests that isolates are clones. However, we identified SNPs segregating in two isolates:

A

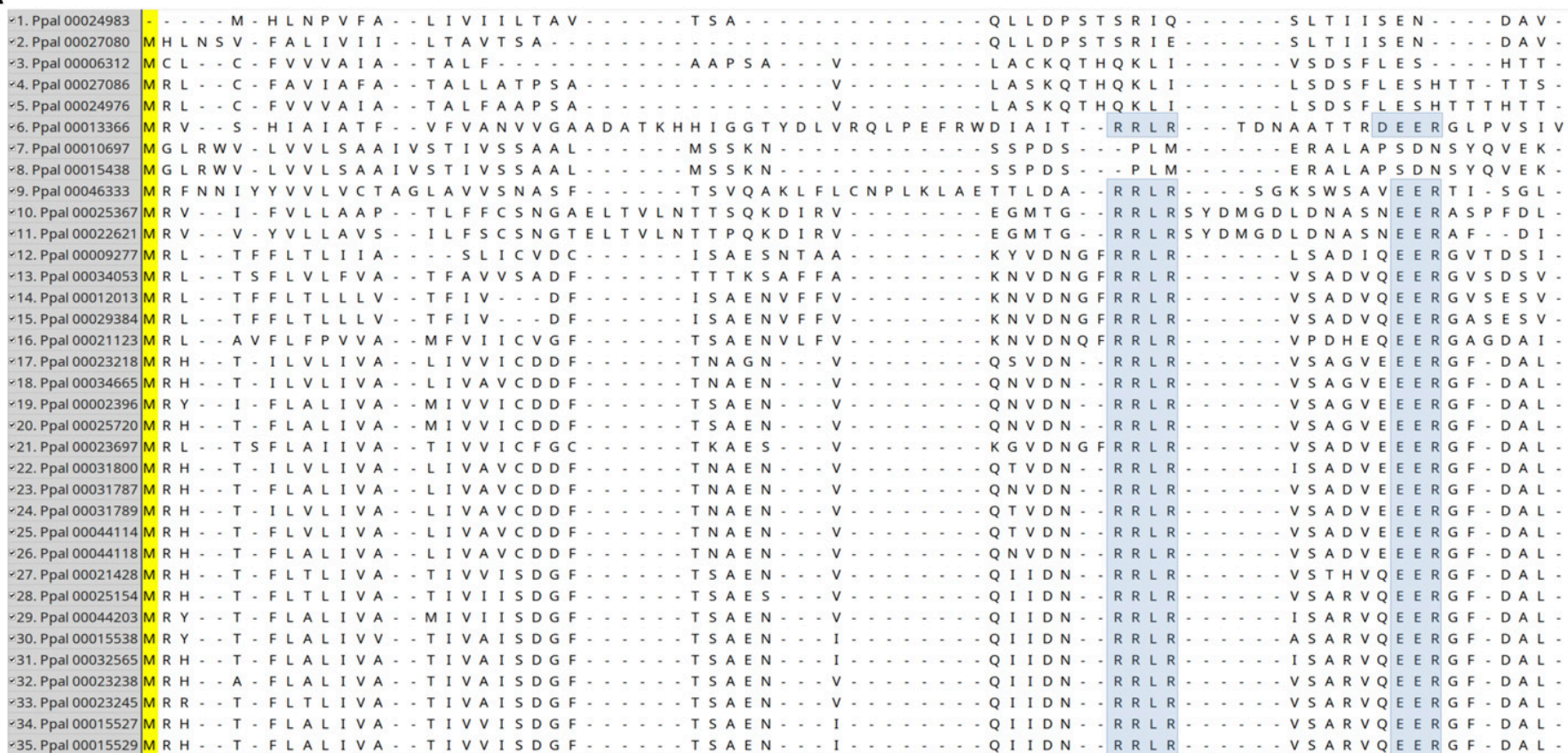

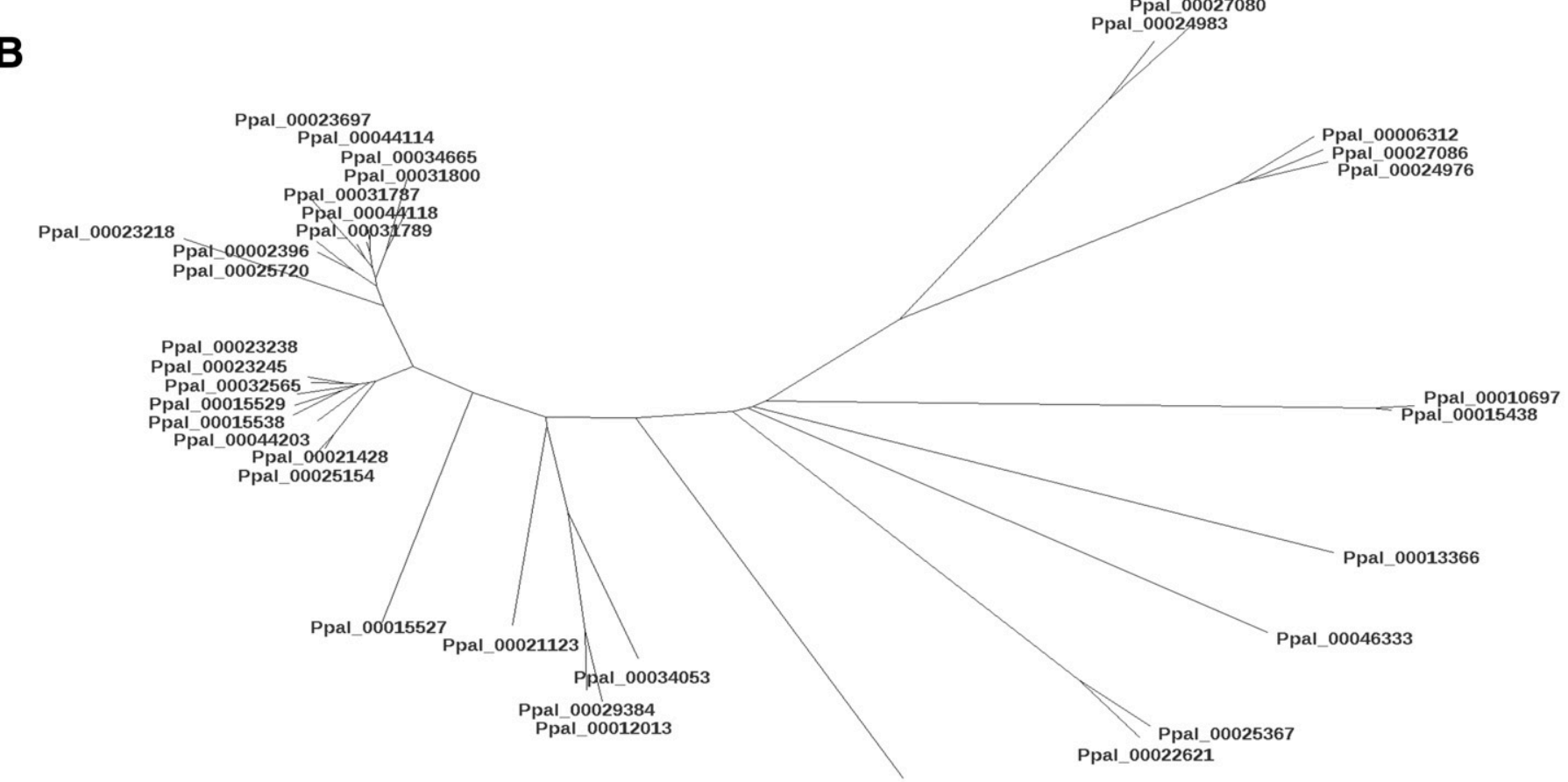

Ppal_00009277

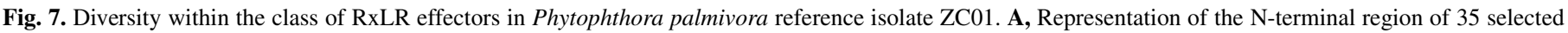

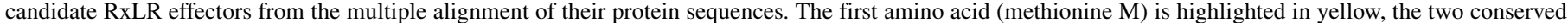
motifs (RRLR and EER) across the sequences are enclosed in blue boxes. B, Neighbor-joining clustering analysis of the same 35 RxLR candidate effectors. 
ZOR01 and ZC02. The first one has been identified as morphologically different for what is expected for P. palmivora. Although this is not the case for isolate $\mathrm{ZC} 02$, we observed that both isolates had a similar disease progression when inoculated on immature oil palm leaflets in the laboratory. Interestingly, isolate ZOR01 originated from a region where bud rot disease is not equally devastating as in other regions of the country, such as palms infected with the pathogen in that region might naturally recover from the disease. Hence, the genetic differences observed between this and other isolates are important and interesting for oil palm researchers, since they may provide insights into the genetic component of the phenotypic variability of the disease within Colombia. This initial result encourages future studies with larger sample sizes and broader phenotypic variability to identify genes related to virulence across the different plantation areas in the country.

The genomic resources provided in this study have supported the understanding of the genetic diversity of P. palmivora associated with the oil palm crop. They can be further exploited as tools for disease resistance studies and other breeding approaches. We report here a high-quality genome assembly for an economically important pathogen worldwide, intraspecies genomic variants genotyped in different strains of P. palmivora, and one of the most significant number of effectors published so far for the species. We expect that these results will aid further investigations on basic genomics and the design of disease control strategies contributing to the research of different groups.

\section{ACKNOWLEDGMENTS}

We thank the members of the Biology and Breeding Research Program and the Phytopathology Research group at Cenipalma for support; the Laboratory of Mycology and Phytopathology (LAMFU) and the HighPerformance Computing Service at Universidad de los Andes, as well as Tatiana García for their contributions.

\section{LITERATURE CITED}

Adhikari, B. N., Hamilton, J. P., Zerillo, M. M., Tisserat, N., Lévesque, C. A., and Buell, C. R. 2013. Comparative genomics reveals insight into virulence strategies of plant pathogenic oomycetes. PLoS One 8:e75072.

Ali, S. S., Shao, J., Lary, D. J., Kronmiller, B. A., Shen, D., Strem, M. D., Amoako-Attah, I., Akrofi, A. Y., Begoude, B. A., Ten Hoopen, G. M., Coulibaly, K., Kebe, B. I., Melnick, R. L., Guiltinan, M. J., Tyler, B. M., Meinhardt, L. W., and Bailey, B. A. 2017. Phytophthora megakarya and Phytophthora palmivora, closely related causal agents of cacao black pod rot, underwent increases in genome sizes and gene numbers by different mechanisms. Genome Biol. Evol. 9:536-557.

Avila-Mendez, K., Rodrigo, Á., Araque, L., and Romero, H. M. 2019. Simultaneous transcriptome analysis of oil palm clones and Phytophthora palmivora reveals oil palm defense strategies. PLoS One 14:e0222774.

Bickhart, D. M., Rosen, B. D., Koren, S., Sayre, B. L., Hastie, A. R., Chan, S., Lee, J., Lam, E. T., Liachko, I., Sullivan, S. T., Burton, J. N., Huson, H. J., Nystrom, J. C., Kelley, C. M., Hutchison, J. L., Zhou, Y., Sun, J., Crisà, A., Ponce de León, F. A., Schwartz, J. C., Hammond, J. A., Waldbieser, G. C., Schroeder, S. G., Liu, G. E., Dunham, M. J., Shendure, J., Sonstegard, T. S., Phillippy, A. M., Van Tassell, C. P., and Smith, T. P. L. 2017. Single molecule sequencing and chromatin conformation capture enable de novo reference assembly of the domestic goat genome. Nat. Genet. 49:643-650.

Bozkurt, T. O., Schornack, S., Banfield, M. J., and Kamoun, S. 2012. Oomycetes, effectors, and all that jazz. Curr. Opin. Plant Biol. 15:483-492.

Bryant, D. M., Johnson, K., DiTommaso, T., Tickle, T., Couger, M. B., Payzin-Dogru, D., Lee, T. J., Leigh, N. D., Kuo, T. H., Davis, F. G., Bateman, J., Bryant, S., Guzikowski, A. R., Tsai, S. L., Coyne, S., Ye, W. W., Freeman, R. M., Jr., Peshkin, L., Tabin, C. J., Regev, A., Haas, B. J., and Whited, J. L. 2017. A tissue-mapped axolotl de novo transcriptome enables identification of limb regeneration factors. Cell Rep. 18:762-776.

Cantarel, B. L., Korf, I., Robb, S. M. C., Parra, G., Ross, E., Moore, B., Holt, C., Sánchez Alvarado, A., and Yandell, M. 2008. MAKER: An easy-to-use annotation pipeline designed for emerging model organism genomes. Genome Res. 18:188-196.

Castiblanco, C., Etter, A., and Aide, M. 2013. Oil palm plantations in Colombia: a model of future expansion. Environ. Sci. Policy 27:172-183.

Drenth, A., and Guest, D. I. (eds.) 2004. Diversity and Management of Phytophthora in Southeast Asia. ACIAR Monograph. 114:7-9.
Eddy, S. R. 2008. A probabilistic model of local sequence alignment that simplifies statistical significance estimation. PLOS Comput. Biol. 4: e1000069.

Evangelisti, E., Gogleva, A., Hainaux, T., Doumane, M., Tulin, F., Quan, C., Yunusov, T., Floch, K., and Schornack, S. 2017. Time-resolved dual transcriptomics reveal early induced Nicotiana benthamiana root genes and conserved infection-promoting Phytophthora palmivora effectors. BMC Biol. 15:39.

Fedepalma. 2018. Statistical yearbook 2017. The oil palm agroindustry in Colombia and the world. Fedepalma, Bogota, Colombia.

Goodwin, S. B., Drenth, A., and Fry, W. E. 1992. Cloning and genetic analysis of two highly polymorphic moderately repetitive nuclear DNAs from Phytophthora infestans. Curr. Genet. 22:107-115.

Grabherr, M. G., Haas, B. J., Yassour, M., Levin, J. Z., Thompson, D. A., Amit, I., Adiconis, X., Fan, L., Raychowdhury, R., Zeng, Q., Chen, Z., Mauceli, E., Hacohen, N., Gnirke, A., Rhind, N., di Palma, F., Birren, B. W., Nusbaum, C., Lindblad-Toh, K., Friedman, N., and Regev, A. 2011. Full-length transcriptome assembly from RNA-seq data without a reference genome. Nat. Biotechnol. 29:644-652.

GreenPalm Sustainability. 2019. GreenPalm Sustainability, Hull, U.K. https:// greenpalm.org/

Kamoun, S., Furzer, O., Jones, J. D., Judelson, H. S., Ali, G. S., Dalio, R. J., Roy, S. G., Schena, L., Zambounis, A., Panabières, F., Cahill, D., Ruocco, M., Figueiredo, A., Chen, X. R., Hulvey, J., Stam, R., Lamour, K., Gijzen, M., Tyler, B. M., Grünwald, N. J., Mukhtar, M. S., Tomé, D. F., Tör, M., Van Den Ackerveken, G., McDowell, J., Daayf, F., Fry, W. E., Lindqvist-Kreuze, H., Meijer, H. J., Petre, B., Ristaino, J., Yoshida, K., Birch, P. R., and Govers, F. 2015. The top 10 oomycete pathogens in molecular plant pathology. Mol. Plant Pathol. 16:413-434.

Kamvar, Z. N., Tabima, J. F., and Grünwald, N. J. 2014. Poppr: An R package for genetic analysis of populations with clonal, partially clonal, and/or sexual reproduction. PeerJ 2:e281.

Kimura, M. 1977. Preponderance of synonymous changes as evidence for the neutral theory of molecular evolution. Nature 267:275-276.

Koren, S., Walenz, B. P., Berlin, K., Miller, J. R., Bergman, N. H., and Phillippy, A. M. 2017. Canu: Scalable and accurate long-read assembly via adaptive $k$-mer weighting and repeat separation. Genome Res. 27:722-736.

Langmead, B., and Salzberg, S. L. 2013. Fast gapped-read alignment with Bowtie 2. Nat. Methods 9:357-359.

Le Fevre, R., O’Boyle, B., Moscou, M. J., and Schornack, S. 2016. Colonization of barley by the broad-host hemibiotrophic pathogen Phytophthora palmivora uncovers a leaf development-dependent involvement of Mlo. Mol. Plant-Microbe Interact. 29:385-395.

Li, H., Handsaker, B., Wysoker, A., Fennell, T., Ruan, J., Homer, N., Marth, G., Abecasis, G., Durbin, R., and 1000 Genome Project Data Processing Subgroup. 2009. The Sequence Alignment/Map format and SAMtools. Bioinformatics 25:2078-2079.

Martin, F. N., Blair, J. E., and Coffey, M. D. 2014. A combined mitochondrial and nuclear multilocus phylogeny of the genus Phytophthora. Fungal Genet. Biol. 66:19-32.

Maurice, S., Montes, M. S., Nielsen, B. J., Bødker, L., Martin, M. D., Jønck, C. G., Kjøller, R., and Rosendahl, S. 2019. Population genomics of an outbreak of the potato late blight pathogen, Phytophthora infestans, reveals both clonality and high genotypic diversity. Mol. Plant Pathol. 20: 1134-1146. 10.1111/mpp.12819

Mohamed Azni, I. N. A., Sundram, S., Ramachandran, V., and Idris, A. 2017. An in vitro investigation of Malaysian Phytophthora palmivora isolates and pathogenicity study on oil palm. J. Phytopathol. 165:800-812.

Nakamura, T., Yamada, K. D., Tomii, K., and Katoh, K. 2018. Parallelization of MAFFT for large-scale multiple sequence alignments. Bioinformatics 34:2490-2492.

Pertea, M., Pertea, G. M., Antonescu, C. M., Chang, T. C., Mendell, J. T., and Salzberg, S. L. 2015. StringTie enables improved reconstruction of a transcriptome from RNA-seq reads. Nat. Biotechnol. 33:290-295.

Petersen, T. N., Brunak, S., von Heijne, G., and Nielsen, H. 2011. SignalP 4.0: Discriminating signal peptides from transmembrane regions. Nat. Methods 8:785-786.

Qiao, X., Li, Q., Yin, H., Qi, K., Li, L., Wang, R., Zhang, S., and Paterson, A. H. 2019. Gene duplication and evolution in recurring polyploidizationdiploidization cycles in plants. Genome Biol. 20:38.

Sarria, G. A., Martínez, G., Varon, F. H., Drenth, A., and Guest, D. I. 2016. Histopathological studies of the process of Phytophthora palmivora infection in oil palm. Eur. J. Plant Pathol. 145:39-51.

Sarria, G. A., Torres, G. A., Aya-Castañeda, H. A., Ariza, J. G., Rodríguez, J., Vélez, D. C., Varón, F. H., and Martínez, G. 2008. Phytophthora sp. is responsible for bud rot in oil palm in Colombia. Palmas. 29:31-41.

Simão, F. A., Waterhouse, R. M., Ioannidis, P., Kriventseva, E. V., and Zdobnov, E. M. 2015. BUSCO: assessing genome assembly and annotation completeness with single-copy orthologs. Bioinformatics 31:3210-3212. 
Tabima, J. F., and Grünwald, N. J. 2019. effectR: An expandable R package to predict candidate RxLR and CRN effectors in oomycetes using motif searches. Mol. Plant-Microbe Interact. 32:1067-1076.

Teixeira da Silva, J. A., and Engelmann, F. 2017. Cryopreservation of oil palm (Elaeis guineensis Jacq.). Cryobiology 77:82-88.

Tello, D., Garcia, R., Escobar-Velásquez, C., Linares-Vásquez, M., and Duitama, J. 2019b. Efficient homolog identification and interactive webbased whole genome alignment [version 1; not peer reviewed]. F1000Research 8(ISCB Comm J):1290 (poster). https://doi.org/10.7490/f1000research. 1117219.1

Tello, D., Gil, J., Loaiza, C. D., Riascos, J. J., Cardozo, N., and Duitama, J. 2019a. NGSEP3: Accurate variant calling across species and sequencing protocols. Bioinformatics 35:4716-4723. https://doi.org/10.1093/bioinformatics/ btz275

Torres, G. A., Sarria, G. A., Martinez, G., Varón, F., Drenth, A., and Guest, D. I. 2016. Bud rot caused by Phytophthora palmivora: A destructive emerging disease of oil palm. Phytopathology 106:320-329.
Torres, G. A., Sarria, G. A., Varón, F. H., Coffey, M. D., Elliott, M. L., and Martínez, G. 2010. First report of bud rot caused by Phytophthora palmivora on African oil palm in Colombia. Plant Dis. 94:1163.

Weiß, C. L., Pais, M., Cano, L. M., Kamoun, S., and Burbano, H. A. 2018. nQuire: A statistical framework for ploidy estimation using next generation sequencing. BMC Bioinformatics 19:122.

Win, J., Chaparro-Garcia, A., Belhaj, K., Saunders, D. G. O., Yoshida, K., Dong, S., Schornack, S., Zipfel, C., Robatzek, S., Hogenhout, S. A., and Kamoun, S. 2012. Effector biology of plant-associated organisms: Concepts and perspectives. Cold Spring Harbor Symp. Quant. Biol. 77: 235-247.

Yang, M., Duan, S., Mei, X., Huang, H., Chen, W., Liu, Y., Guo, C., Yang, T., Wei, W., Liu, X., He, X., Dong, Y., and Zhu, S. 2018. The Phytophthora cactorum genome provides insights into the adaptation to host defense compounds and fungicides. Sci. Rep. 8:1. 10.1038/s41598-018-24939-2

Yang, Z. 2007. PAML 4: A program package for phylogenetic analysis by maximum likelihood. Mol. Biol. Evol. 24:1586-1591. 